\title{
A Survey on Offline Signature Recognition and Verification Schemes
}

\author{
Jyoti Singh $^{1}$, Dr. Manisha Sharma ${ }^{2}$ \\ ${ }_{1,2}$ Bhilai Institute of Technology, Durg (C.G.)
}

\begin{abstract}
Signature has been a distinguishing biometric feature through ages. They are extensively used as a means of personal verification; therefore an automatic verification system is needed. Even today thousands of financial and business transactions are being authorized via signatures. Signature verification finds its application in a large number of fields starting from online banking, passport verification systems to even authenticating candidates in public examinations from their signatures. This paper represents a brief survey on various off-line signature recognition \& verification schemes.
\end{abstract}

Categories and Subject Descriptors

1.4 Image Processing and Computer Vision

General Terms: Systems, Forgeries, Methods, Skilled, Performance.

Keywords: Off-line Signature verification, on-line signature verification, biometrics, authentication systems

\section{Introduction}

Signature verification is not only a major area of research in the field of image processing and pattern recognition, but also widely used in the fields of finance, access control, contractual matters and security. A signature verification system and various techniques for signature verification are categorized into two separate classes, namely On-line method and Off-line signature verification method. On-line signature verification focuses on capturing and analyzing the signature in real time, as the person is signing it. Off-line signature verification deals with analyzing images of a person's signature.

On-line signature verifications system records the motion of the stylus (which is also part of the sensor) while the signature is produced, and includes location, and possibly velocity, acceleration and pen pressure, as functions of time. Off-line method that uses an optical scanner to obtain handwriting data written on paper deals with a 2-Dimage of the signature. Off-line method is complex due to the absence of stable dynamic characteristics. The non-repetitive nature of variation of the signatures, because of age, illness, geographic location and perhaps to some extent the emotional state of the person, accentuates the problem.

This paper is organized as follows: Section II discusses the signature verification concept, Section III introduces different types of forgeries, Section IV introduces different methods of signature verification, Section $\mathrm{V}$ introduces a comparison of different approaches, and Section VI concludes the paper and shows scope of future work.

\section{Signature Verification Concept}

A signature is any written specimen in a person's own handwriting meant to be used for identification. A signature verification (SV) system authenticates the identity of any person, based on an analysis of his/her Signature through a set of processes which differentiates a genuine signature from a forgery signature. The precision of signature verification systems can be expressed by two types of error: the percentage of genuine signatures rejected as forgery which is called False Rejection Rate (FRR); and the percentage of forgery signatures accepted as genuine which is called False Acceptance Rate (FAR). While dealing with any signature verification system, we consider FRR and FAR as its performance evaluation parameters.

\section{Types Of Forgeries}

There are three kinds of forgeries -Random, Casual and Skilled.

(a) Random Forgery: The random forgery is written by the person who doesn't know the shape of the original signature.

(b) Casual Forgery: The second, called Casual forgery is represented by a signature sample, written by the person who knows the shape of the original signature without much practice.

(c) Skilled Forgery: The third type called the skilled forgery, represented by a suitable imitation of the genuine signature model. 
But based on the various skilled levels of the forgeries, it can also be divided into six different subsets: (a) Random Forgery (b) Casual Forgery (c) Simulated Forgery (d) Unskilled (e) Targeted Forgery and (f) Skilled Forgery

\section{Methods For Off-Line Signature Verification}

Many methods have been developed for Signature Verification. Some of the convenient approaches and optimized methods are discussed below:

Justino, Bortolozzi and Sabourin proposed an off-line signature verification system using Hidden Markov Models to detect random, casual, and skilled forgeries. Three features: a pixel density feature, a pixel distribution feature and an axial slant feature are extracted from a grid segmentation scheme. A database of 1600 genuine signatures is used to determine the optimal codebook size for detecting random forgeries. Signatures of 60 writers with 40 training signatures, 10 genuine test signatures, 10 casual forgeries, and 10 skilled forgeries per writer is used in another data set for experimentation. A False Acceptance rate of $2.83 \%$ is obtained and a False Rejection rate of $1.44 \%, 2.50 \%$, and $22.67 \%$ are obtained for random, casual, and skilled forgeries, respectively.

Mehdi Dehghan, Karim Faez \& Mahmood Fathi proposed an offline signature verification system using shape descriptors \& multiple neural networks. The verification was based on the signature shape descriptors such as the skeleton, upper and lower envelopes of the signature and high pressure region of the signatures. Multiple multilayer perceptron neural network module was use to make a decision of verification using fuzzy integral voter. A multiple multi layer perceptron was selected as the signature classifier for each different signature shape descriptor. The networks were trained by a modified backpropagation algorithm. The neural network was trained to approximate fuzzy set membership. A database of 1000signatures from 50 persons was created with random and skilled forgeries. An accuracy of $96 \%$ for simple and $90 \%$ for skilled was obtained. The system verified the signatures with an overall accuracy of $98 \%$.

H. Baltzakisa, N. Papamarkos developed a signature verification technique based on a two-stage neural network classifier. The proposed system was based on global, grid and texture features. For each one of these feature sets a special two stage Perceptron OCON (one-class-one-network) classification structure was implemented. In the first stage, the classifier combines the decision results of the neural networks and the Euclidean distance obtained using the three feature sets. The results of the first-stage classifier feed a secondstage radial base function (RBF) neural network structure, which makes the final decision. A database of about 2000 signatures were taken from 115 persons (1525 signatures from each).For training the system, two subsets, taken from the master set, of about 1000 and 500 signatures were used. The first subset (TRS1) was used to train the first-stage classifier while the second subset (TRS2) was used to train the second-stage classier. The performance of the system was checked by the use of the remaining subset (TS) of 500 signatures. A FAR of $9.81 \%$ FRR of $3 \%$ and an overall efficiency of $90.09 \%$ was achieved.

Ismail et al. developed an off-line signature identification method. A data base of 2400 signature images is considered. Chain code feature extraction is used to represent a boundary by a connected sequence of straight-line segments of specified length and direction. Seven different types of distance measure were used based on feature vectors derived from Eigen-signatures. The highest accuracy of $96.2 \%$ is obtained with the Manhattan distance measure.

Emre Özgündüz, Tülin Şentürk and M. Elif Karslıgil proposed an offline signature verification system using Support vector machine. The verification and recognition system used the global, directional and grid features of signatures. Support Vector Machine (SVM) was used to verify and classify the signatures and a classification ratio of 0.95 was obtained. SVM was a new learning method introduced by V. Vapnik et al. SVMs learn linear threshold functions, in nonlinear case; they can be used to learn polynomial classifiers, radial basis function (RBF) nets, multi layer perceptron and the like by applying appropriate kernel functions. In the experiments, a comparison between SVM and ANN is performed. Using a SVM with RBF kernel, an FRR of $0.02 \%$ and an FAR of $0.11 \%$ are obtained. Whereas the ANN, trained with the Back propagation algorithm, provided an FRR of $0.22 \%$ and an FAR of \%0.16. In both experiments, skilled forgeries are used to train the classifier.

Stephane Armand, Michael Blumenstein and Vallipuram Muthukkumarasamy proposed an Off-line Signature Verification based on the Modified Direction Feature. The system uses a database of 2106 signatures. MDF employs a hybrid of two feature extraction techniques, Direction Feature (DF) and the Transition Feature (TF).DF extracted direction transitions (DT), based on the replacement of the foreground pixels by their direction values. The feature vector was extracted by zoning and computing the most representative direction values in a given zone.TF recorded the locations of the transitions (LTs) between foreground (1s) and background (0s) in binary digital images. The image was traversed from following directions: left to right, right to left, top to bottom and bottom to top. Each time a change from ' 0 ' to ' 1 ' or from ' 1 ' to ' 0 ' occurred, the ratio between the location of the transition and the length/width of the image traversed was recorded as a feature. An averaging algorithm was used to obtain a feature vector of appropriate size in order to decrease the training/classification time. A centroid feature and a trisurface feature were also used for enhancing the accuracy 
of the result. Two neural network classifiers were used to classify the signatures: the resilient backpropagation (RBP) neural network and the Radial Basis Function RBF) network. For RBP verification rate of $86.08 \%$ and for RBF a verification rate of $89.61 \%$ was achieved. The highest accuracy obtained was $91.12 \%$.

Nguyen et al. presented an off-line signature verification system based on global features. In their paper, the combination of the Modified Direction Feature (MDF) with three global features: Feature from Energy Information, Maxima Feature, and Ratio Feature is reported. MDF feature extraction technique employs the location of transitions from background to foreground pixels and the direction at transitions in the vertical and horizontal directions of the boundary representation of an object. At each transition, the Location of the Transition (LT) and the Direction Transition (DT) values are recorded. A database of 12 genuine specimens and 400 random forgeries are taken from a publicly available database. The Support Vector Machine (SVM) classifier obtained an average error rate (AER) of $17.25 \%$.

Bradley Schafer, Serestina Viriri presented an offline signature verification system based on global features and transition features. In this paper a database of 2106 signatures was used. To train the system, a subset of this database was taken comprising of 15 genuine samples taken from each of the 30 different individual's signatures. The 15 genuine signatures from each person would then have all the features extracted from each one. The features for all 15 signatures would then be averaged to form one centroid feature vector. This centroid feature vector is what is then stored in template file that is used as a reference for all signatures belonging to that group. When a claimed signature is entered into the system, it is compared against the centroid feature vectors that are obtained to ascertain whether it is a genuine signature or a forged signature. During the testing phase, two approaches were tested. During testing, a claimed signature is compared against our template file using the Euclidean distance and if it is below a certain threshold value, then the signature is declared valid, otherwise it is a forgery. The first approach is to use a global threshold to do all the comparisons against. Using the global threshold correct classification rate of $73 \%$ and a false acceptance rate of $18.5 \%$ were obtained. Using the second approach, the localized threshold, a correct classification rate of $84.1 \%$ and a false acceptance rate of $17.8 \%$ was obtained.

Hanno Coetzer and Robert Sabourin proposed a human-centric system, which exploited the synergy between human and machine capabilities. The combination strategy was based on techniques in receiver operating characteristics (ROC) analysis. This paper investigated the possibility of enhancement of the performance of an existing off-line signature verification system by also utilizing proficient human operators. The feature extraction was based on the calculation of the Radon transform of a signature image and each writer's signature is modelled by a ring-structured HMM. A data set of 765 test signatures from 51 writers was created. The performance of 23 human classifiers and that of a hidden Markov model-based (HMM-based) classifier, in ROC space was recorded the best possible performance of an existing HMM-based classifier was $87.0 \%$, and that of a human of $91.0 \%$. The best performance of $96.5 \%$ is attainable by combining the outputs of an HMM-based classifier and a human classifier.

Deng proposed a system that used a closed contour tracing algorithm to represent the edges of each signature with several closed contours. The curvature data of the traced closed contours were decomposed into multi-resolution signals using wavelet transforms. The zero crossings corresponding to the curvature data were extracted as features for matching. A statistical measurement was devised to decide systematically which closed contours and their associated frequency data were most stable and discriminating. Based on these data, the optimal threshold value which controls the accuracy of the feature extraction process was calculated. Matching was done through dynamic time warping. Experiments were conducted independently on two data sets, one consisting of English signatures and the other consisting of Chinese signatures. For each experiment, 25 writers are used with 10 training signatures, 10 genuine test signatures, 10 skilled forgeries, and ten casual forgeries per writer. When only the skilled forgeries are considered, AERs of $13.4 \%$ and $9.8 \%$ are reported for the respective data sets. When only the casual forgeries are considered, AERs of $2.8 \%$ and $3.0 \%$ are reported.

Abhay Bansal, Bharat Gupta, Gaurav Khandelwal, and Shampa Chakraverty proposed an Offline Signature Verification Using Critical Region Matching. The system was designed to detect the skilled forgeries and was focused on the extraction of the critical regions which are more prone to mistakes and matched them following a modular graph matching approach. The method includes critical points extraction, critical region extraction, and formulation of signature verification problem as a graph matching problem. A database of 240 signatures 5 genuine signatures of each of 76 persons was taken. For each of the 76 persons, 4 semi-skilled and 4 skilled forgeries were collected. For semi-skilled forgeries an accuracy of $95.69 \%$ and for skilled forgeries an accuracy of $89.09 \%$ was obtained.

Oliveira et al. developed an off-line signature verification system based on the Writer-Independent approach. Receiver Operating Characteristic (ROC) curves is used to improve the performance of the system. ROC graphs are two dimensional graphs in which true positive rate (TPR) and false positive rate (FPR) are plotted on the $\mathrm{Y}$ and $\mathrm{X}$ axis respectively. They used a two-fold technique. At first, different fusion strategies are analysed based on the ROC. Next, the result of the first stage is further improved by combining the classifiers without the need of joint training. They used two sets of data (160 genuine signatures, 40 forgery signatures and 
1200 genuine, 300 forgery signatures). Support Vector Machine is used as a classifier and they obtained $91.80 \%$ as the highest recognition rate.

Kaewkongka, Chamnongthai and Thipakom proposed a method of off-line signature recognition by using Hough transform to detect stroke lines from signature image. The Hough transform was used to extract the parameterized Hough space from signature skeleton as unique characteristic feature of signatures. In the experiment, the Back Propagation trained Neural Network was used as a tool to evaluate the performance of the proposed method. The system was tested with 70 test signatures from different persons. The experimental results reveal the recognition rate $95.24 \%$.

Fang proposed a system that was based on the assumption that the cursive segments of forged signatures are generally less smooth than that of genuine ones. Two approaches were proposed to extract the smoothness feature: a crossing method and a fractal dimension method. The smoothness feature is then combined with global shape features. Verification was based on a minimum distance classifier. An iterative leave-one-out method was used for training and for testing genuine test signatures. A database with 55 writers was used with 24 training signatures and 24 skilled forgeries per writer. An AER of $17.3 \%$ was obtained.

Abbas used a back propagation neural network prototype for the offline signature recognition. He used feed forward neural networks and three different training algorithms Vanila, Enhanced and batch were used. he reported FAR between the range of $10-40 \%$ for casual forgeries. A neuro-fuzzy system was proposed by Hanmandlu, they compared the angle made by the signature pixels are computed with respect to reference points and the angle distribution was then clustered with fuzzy c - means algorithm. Back propagation algorithm used for training neural network. The system reported FRR in the range of 5-16\% with varying threshold.

Ismail et al. proposed an off-line Arabic signature recognition and verification technique. In their paper, a system of two separate phases for signature recognition and verification is developed. In the first phase some features based on Translation, circularity feature, image enhancement, partial histogram, centres of gravity, global baseline, thinning etc. are extracted. In the second phase some more features are also extracted such as Central line features, Corner line features Central circle features, Corner curve features and Critical point features. A set of signature data consisting of 220 genuine samples and 110 forged samples is used for experimentation. They obtained a $95.0 \%$ recognition rate and $98.0 \%$ verification.

\section{Performance Evaluation Of Different Approaches With Result}

Evaluation parameters for any signature verification system are FAR and FRR. The performances of different important methods with associated results are shown in Table 1. In table 1, accuracy (\%) denotes the average Identification rate of different databases. In the table 1 the FAR is shown for all different forgeries. The results shows that very good high accuracy is still lacking from existing systems and hence further work is required in this area.

FRR- False Rejection Rate

FAR- False Acceptance Rate

Table1. Evaluation of performances of diff. methods.

\begin{tabular}{|l|l|l|l|l|}
\hline $\begin{array}{l}\text { Serial } \\
\text { No. }\end{array}$ & Approach & FRR & FAR & $\begin{array}{l}\text { Accuracy } \\
(\%)\end{array}$ \\
\hline 1. & $\begin{array}{l}\text { SVM based } \\
\text { approach }\end{array}$ & 4.83 & 5.30 & 91.9 \\
\hline 2. & $\begin{array}{l}\text { Virtual Support } \\
\text { vector machine }\end{array}$ & 16.00 & 13.00 & - \\
\hline 3. & $\begin{array}{l}\text { Global Feature } \\
\text { Based }\end{array}$ & 5.40 & 4.60 & - \\
\hline 4. & $\begin{array}{l}\text { Enhanced } \\
\text { modified } \\
\text { direction } \\
\text { feature }\end{array}$ & 2.88 & 1.71 & 91.21 \\
\hline 5. & $\begin{array}{l}\text { Based on } \\
\text { Neural network }\end{array}$ & 15.00 & 3.00 & - \\
\hline 6. & $\begin{array}{l}\text { Hidden Markov } \\
\text { Model and } \\
\text { Cross- } \\
\text { Validation }\end{array}$ & 00.64 & 11.70 & - \\
\hline 7. & $\begin{array}{l}\text { Signature } \\
\text { feature } \\
\text { Correspondence }\end{array}$ & 6.30 & 8.20 & 91.80 \\
\hline Contour & 13.20 & 11.60 & 86.90 \\
\hline
\end{tabular}




\begin{tabular}{|l|l|l|l|l|}
\hline & Method & & & \\
\hline 9. & $\begin{array}{l}\text { Based on ROC } \\
\text { analysis }\end{array}$ & - & - & 96.50 \\
\hline 10. & $\begin{array}{l}\text { Two-stage } \\
\text { neural } \\
\text { network } \\
\text { classifier }\end{array}$ & 09.81 & 03.00 & 80.81 \\
\hline
\end{tabular}

\section{Conclusion And Feature Scope Of Work}

This paper presents a brief survey of the recent works on off-line signature recognition \& verification. Different existing approaches are discussed and compared along with their FAR, FRR and accuracies.

As we could observe that lots of work has already been done in the field of signature verification, there are still many challenges in this research area. The non-repetitive nature of variation of the signatures, because of age, illness, geographic location and perhaps to some extent the emotional state of the person, accentuates the problem. Another problem associated with this area is, for security reasons, it is not easy to make a signature dataset of real documents (such as banking documents, for example) available to the signature verification community. Publicly available signature datasets of real documents would make it possible for researchers to achieve a better performance in this field.

The accuracy obtained so far from the available systems is not very high and more research on Off-line Signature verification \& On-line Signature verification is required.

\section{References}

[1] P. Deng, H. Yuan Mark Liao \& H. Tyan, "Wavelet` Based Off-line Signature Recognition System", Proceedings 5th Conference on Optical Character Recognition and Document Analysis, Beijing, China., 1996.

[2] Mehdi Dehghan, Karim Faez \& Mahmood Fathi "Offline signature verification system using shape descriptors \& multiple neural networks", IEEE, 1997.

[3] T. Kaewkongka, K. Chamnongthai, B. Thipakom, "Off-Line Signature Recognition using parameterized Hough Transform ", ISSPA Brisbane, Australia, 1999.

[4] H. Baltzakis, N. Papamarkos, "A new signature verification technique based on a two-stage neural network classifier", Engineering Applications of Artificial Inteligence ,2001.

[5] J Edson, R. Justino, F. Bortolozzi and R. Sabourin, "An off-line signature verification using HMM for Random, Simple and Skilled Forgeries", 2001.

[6] J Edson, R. Justino, F. Bortolozzi and R. Sabourin, "The Interpersonal and Intrapersonal Variability Influences on Off-line Signature Verification Using HMM", Brazilian Symp, Computer Graphics and Image Processing, 2002.

[7] Martinez, L.E., Travieso, C.M, Alonso, J.B., and Ferrer, M. Parameterization of a forgery Handwritten Signature Verification using SVM. IEEE, International Carnahan Conference on Security Technology, 2004.

[8] Emre Özgündüz, Tülin Şentürk and M. Elif Karslıgil "Offline Signature verification and Recognition by Support Vector Machine", EUSIPCO, 2005.

[9] R. Abbas and V. Ciesielski, “A Prototype System for Offline Signature Verification Using Multilayered Feed forward Neural Networks", 1995.

[10] L. S. Oliveira, E. Justino, and R. Sabourin, “Off-line signature verification using writer-independent approach”, IJCNN, 2007.

[11] Hanno Coetzer, Robert Sabourin “A Human-Centric Off-Line Signature Verification System”, IEEE, ICDAR, 2007.

[12] Abhay Bansal, Bharat Gupta, Gaurav Khandelwal, and Shampa Chakraverty "Offline Signature Verification Using Critical Region Matching”, International Journal of Signal Processing, Image Processing and Pattern, 2009.

[13] M.A. Ismail, Samia Gad, "Off-line Arabic Signature Recognition and Verification", 2000.

[14] L. S. Oliveira, E. Justino, and R. Sabourin, "Off-line signature verification using writer-independent approach”, IJCNN, 2007.

[15] P. Deng, H. Yuan Mark Liao \& H. Tyan, "Wavelet Based Off-line Signature Recognition System”, Beijing, China, 1996.

[16] Vu Nguyen, Michael Blumenstein, Vallipuram Muthukkumarasamy, Graham Leedham. "Off Line Signature Verification using Enhanced Modified Direction Features in conjuction with Neural Classifiers and Support Vector Machine”, ICDAR, 2007.

[17] V A Bharadi, H B Kekre "Off-Line Signature Recognition Systems", International Journal of Computer Applications, 2010.

[18] Neeraj Shukla, Dr. Madhu Shandilya "Invariant Features Comparison in Hidden Markov Model and SIFT for Offline Handwritten Signature Database”, International Journal of Computer Applications, 2010.

[19] Manasjyoti Bhuyan, Kandarpa Kumar Sarma and Hirendra Das", Signature Recognition and Verification using Hybrid Features and Clustered Artificial Neural Network (ANN) s", International Journal of Electrical and Computer Engineering, 2010.

[20] O.C Abikoye, M.A Mabayoje, R. Ajibade Offline "Signature Recognition \& Verification using Neural Network", International Journal of Computer Applications, 2011.

[21] Minal Tomar and Pratibha Singh "A Directional Feature with Energy based Offline Signature Verification Network" International Journal on Soft Computing (IJSC), 2011.

[22] B. Majhi, Y. Reddy, D. Babu, "Novel Features for Off-line Signature Verification", International Journal of Computers Communications \& Control, 2006.

[23] H B kekre, V.A. Bharadi, "Specialized Global Features for Off-line Signature Recognition", Annual National Conference on Biometrics RFID and Emerging Technologies for Automatic Identification, 2009.

[24] V A Bharadi, H B kekre, "Off-line Signature Recognition Systems", International Journal of Computer Applications, 2010.

[25] B. Kovari, Z. Kertesz, A. Major, "Off-Line Signature Verification Based on Feature Matching", International Conference on Intelligent Engineering Systems, 2007. 